\title{
Screening for pulmonary tuberculosis in HIV-infected individuals: AIDS Clinical Trials Group Protocol A5253
}

\author{
S. Swindells ${ }^{\star}$, L. Komarow ${ }^{\dagger}$, S. Tripathy ${ }^{\ddagger}$, K. P. Cain $§$, R. R. MacGregorף, J. M. Achkar ${ }^{\#}$, A.

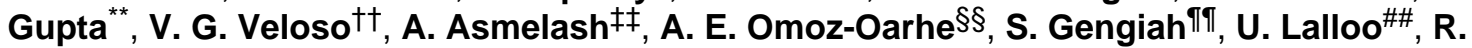 \\ Allen $^{* \star \star}$, C. Shiboski ${ }^{\dagger \dagger \dagger}$, J. Andersen ${ }^{\dagger}$, S. S. Qasba ${ }^{\ddagger \ddagger \ddagger}$, and D. K. Katzenstein ${ }^{\S \S}$ for the AIDS \\ Clinical Trials Group 5253 Study Team
}

"University of Nebraska Medical Center, Omaha, Nebraska †Harvard School of Public Health, Boston, Massachusetts, USA $¥$ National AIDS Research Institute, Pune, India §Centers for Disease Control and Prevention, Atlanta, Georgia IUniversity of Pennsylvania, Philadelphia, Pennsylvania \#Albert Einstein College of Medicine, Bronx, New York "Johns Hopkins University, Baltimore, Maryland, USA †tInstituto de Pesquisa Clinica Evandro, Rio de Janeiro, Rio de Janeiro, Brazil ${ }^{\ddagger}$ Scottish Livingstone Hospital, Molepole §§Princess Marina Hospital, Gaborone, Botswana "TICenter for the AIDS Programme of Research in South Africa, Durban \#\#University of KwaZulu-Natal, Durban, South Africa ${ }^{* * *}$ AIDS Clinical Trials Group Operations Center, Silver Spring, Maryland t+tUniversity of California San Francisco, San Francisco, California $\ddagger \neq \neq$ Montgomery County Health Department, Silver Spring, Maryland $\$ \S \S S t a n f o r d$ University Medical Center, Stanford, California, USA

\section{Abstract}

BACKGROUND—Improved tuberculosis (TB) screening is urgently needed for human immunodeficiency virus (HIV) infected patients.

\begin{abstract}
METHODS-An observational, multicountry, cross-sectional study of HIV-infected patients to compare a standardized diagnostic evaluation (SDE) for TB with standard of care (SOC). SOC evaluations included TB symptom review (current cough, fever, night sweats and/or weight loss), sputum Ziehl-Neelsen staining and chest radiography. SDE screening added extended clinical signs and symptoms and fluorescent microscopy (FM). All participants underwent all evaluations. Mycobacterium tuberculosis on sputum culture was the primary outcome.
\end{abstract}

Correspondence to: Susan Swindells, Department of Internal Medicine, University of Nebraska Medical Center, 988106 Nebraska Medical Center, Omaha, NE 68198-8106, USA. Tel: (+1) 402559 5392. Fax: (+1) 402553 5527. sswindells@ unmc.edu.

Trial Registration: ClinicalTrials.gov Trial Registration Number: NCT00865826 at http://clinicaltrials.gov/

Sites and contributors: R R Gangakhedkar and A Risbud, International Clinical Trials Unit Pune, India (Site 11601) Clinical Trials Unit (CTU) Grant U01 AIO69417-05; S Selebaleng and R Molefe-Molepolole Prevention \& Treatment Center, Botswana (Site 12702) CTU Grant U01 AI069456; K Kooreng and M Raesi, Gaborone Prevention \& Treatment Center, Botswana (Site 12701) CTU Grant U01 AI069456; K Naidoo, CAPRISA eThekwini Clinical Research Site (CRS), Durban, South Africa (Site 31422) CTU Grant 5U01AI069469-05; S Pillay, Durban International Clinical Trials Unit, South Africa (Site 11201) CTU Grant 5U1A1069426-05; D Kadam and V Mave, Byramjee Jeejeebhoy Medical College CTS, Pune, India (Site 31441) CTU Grant U01 AI069497; P Ive and M Hero, Wits HIV CRS, Johannesburg, South Africa (Site 11101) CTU Grant 5UM1 AI069463-05; L Mohapi and R Lourens, Soweto ACTG CRS, South Africa (Site 12301) CTU Grant U01AI69453; V Gs Veloso and M C da Silva Lourenço, Instituto de Pesquisa Clinica Evandro Chagas CRS, Brazil (Site 12101) CTU Grant U01AI069476; A Mwafongo and A Moses, University of North Carolina Project, Kamuzu Central Hospital, Lilongwe, Malawi (Site 12001) CTU Grant U01 AI069518; P Segura and C Ganoza, Barranco CRS, Lima, Peru (Site 11301); C Ganoza and P Segura, Investigaciones Medicas en Salud, Lima, Peru (Site 11302) CTU Grant U01AI069438; G Kadzirange and R Mahachi, UZ Parirenyatwa Clinical Research Site, Harare, Zimbabwe (Site 30313), CTU Grant U01 A1069436. 
RESULTS-A total of 801 participants enrolled from Botswana, Malawi, South Africa, Zimbabwe, India, Peru and Brazil. The median age was 33 years; $37 \%$ were male, and median CD4 count was 275 cells $/ \mathrm{mm}^{3}$. Thirty-one participants (4\%) had a positive culture on LöwensteinJensen media and $54(8 \%)$ on MGIT. All but one positive culture came from sub-Saharan Africa, where the prevalence of TB was 54/445 (12\%). SOC screening had 54\% sensitivity (95\%CI 4067 ) and $76 \%$ specificity (95\% CI 72-80). Positive and negative predictive values were $24 \%$ and $92 \%$. No elements of the SDE improved the predictive values of SOC.

CONCLUSIONS-Symptom-based screening with smear microscopy was insufficiently sensitive. More sensitive diagnostic testing is required for HIV-infected patients.

\section{Keywords}

tuberculosis; diagnostics; HIV infection; sensitivity; specificity

Millions of human immunodeficiency virus (HIV) infected individuals will initiate antiretroviral treatment (ART) in settings where tuberculosis (TB) is endemic. ${ }^{1}$ The increasing prevalence of HIV infection has resulted in a 3- to 10-fold increase in active TB in settings where an estimated 30-60\% of adults are infected with TB, the leading opportunistic infection associated with HIV infection. ${ }^{2}$ In many countries in Africa, more than two thirds of active TB cases identified are in HIV-infected persons, and screening for TB has recently become standard of care before the initiation of antiretroviral therapy (ART). ${ }^{3}$ Strategies to optimize screening, diagnosis and treatment of TB are integral to the public health treatment of HIV. ${ }^{4}$

The incidence and prevalence of active TB in the HIV-infected populations vary widely in different settings, and detection of TB may depend on the underlying prevalence of TB and the diagnostic methods employed. The gold standard for the diagnosis of pulmonary TB is sputum culture positivity. ${ }^{5}$ However, the costs and complexities of routine cultures for TB as well as the delay in receiving results limit the widespread use of sputum culture for diagnosis. New diagnostic strategies using the more sensitive polymerase chain reaction (PCR) amplification of Mycobacterium tuberculosis DNA have recently been introduced, but these may be too expensive for widespread use in resource limited settings. ${ }^{6,7}$ Simple, lower-cost diagnostic strategies are needed to implement effective treatment of TB in the context of epidemic HIV infection and increasing multidrug resistance. ${ }^{8,9}$

Among HIV-infected populations, case finding based on standard sputum smear results in missed diagnoses and delay in initiating anti-tuberculosis treatment. ${ }^{10}$ Extended symptom screening may increase sensitivity, particularly in patients with a CD4+ cell counts of 2350 / $\mathrm{mm}^{3}$ or more. ${ }^{11}$ The incidence of active TB disease increases as CD4+ cell numbers decline, and some of the cardinal symptoms of TB--cough, fever, weight loss, and night sweats--may also result from progressive HIV infection and opportunistic infections. Standard recommendations for laboratory screening and diagnostic algorithms for TB have been suggested by the World Health Organization (WHO), and their performance examined in a meta-analysis of studies in the setting of HIV infection. ${ }^{12}$ Absence of all of current cough, fever, night sweats and weight loss had a negative predictive value (NPV) of 90-97\%, depending on the local prevalence of TB. This screening is therefore recommended in highburden settings to identify people living with HIV in need of further diagnostic assessment for TB, and to facilitate scale-up of isoniazid preventive therapy. ${ }^{13}$

The current study was undertaken to prospectively assess the performance of WHO guidance in diverse international HIV-infected populations with varying community burdens of TB. The study was designed and conducted to test the hypothesis that a standardized clinical evaluation for TB will lead to improved identification in HIV-infected participants 
who are not TB suspects and not currently receiving ART, as compared to standard of care (SOC) screening.

\section{METHODS}

\section{Study design and participants}

The AIDS Clinical Trials Group (ACTG) A5253 study was an observational, prospective, cross-sectional study designed to construct a standardized diagnostic evaluation (SDE) that improves the diagnosis of pulmonary TB compared to SOC TB screening in HIV-infected adults not currently receiving ART and not suspected to have TB. For the purposes of the analysis, SOC screening algorithm components were cough, fever, weight loss and/or night sweats occurring in the previous 30 days, Ziehl-Neelsen (ZN) sputum smear and chest radiography (CXR) for non-pregnant participants. SDE screening included additional symptoms and clinical signs, and sputum smear using fluorescent microscopy (FM). Other screening included standardized questions about gastrointestinal, genitourinary, neurological and dermatological symptoms. A targeted physical examination was performed for height, weight, axillary or oral temperature, pulse rate, respiration rate and blood pressure, Karnofsky performance status, oral mucosal examination, examination for lymphadenopathy, examination of the chest, abdomen, skin, and a neurologic examination (detailed in Appendices A and B).* All participants underwent all evaluations.

Participants were recruited between 5 February and 30 November 2010. The primary objective was to construct an SDE that increased identification of participants with active pulmonary TB without sacrificing specificity, in comparison to SOC. Active pulmonary TB was defined as one or more sputum samples culture-positive for M. tuberculosis on Löwenstein-Jensen (LJ) medium or in $\mathrm{BACTEC}^{\mathrm{TM}}$ MGIT $^{\mathrm{TM}}$ culture (BD, Sparks, MD, USA), or both. Secondary objectives reported here were: 1) to characterize the prevalence of $\mathrm{TB}$ in symptomatic and asymptomatic individuals, 2) to characterize the rates of TB drug resistance, and (3) to assess the incremental benefits of the screening evaluations over a range of $\mathrm{CD} 4+$ cell counts.

Participants were recruited from 11 ACTG clinical trials units, which are out-patient clinics mostly located in academic health centers. Sites were required to be in a country with a TB prevalence of $\geq 60$ per 100000 population, and to have a TB laboratory participating in an external quality assurance program under the supervision of the Division of AIDS. ${ }^{14}$ Inclusion criteria were documented HIV-1 infection, age $\geq 13$ years, and ability and willingness to provide informed consent. Exclusion criteria were receipt of ART within 90 days, diagnosis of active TB within 90 days, and current or recent receipt of medications with anti-tuberculous activity. Eligible participants were enrolled consecutively.

The Institutional Review Boards or Ethics Committees of the participating institutions unconditionally approved the study, and each participant provided written informed consent.

\section{Study assessments}

In addition to medical history and physical examination, three individual sputum specimens were requested from each participant. Specimens could be collected using any combination, such as three within 1 day, three within 2 days, or two at the site and one at home within 2 days. ${ }^{15}$ Participants were asked to expectorate sputum, and sites used induction facilities, if available, for those unable to expectorate.

Sputum was treated with $1 \%$ sodium chloride/ $2 \%$ sodium hydroxide for decontamination and digestion following published guidelines. ${ }^{16}$ Smears were prepared using techniques recommended by the WHO or the International Union Against Tuberculosis and Lung 
Disease. ${ }^{17,} 18$ All specimens were examined by light microscopy of at least 100 fields with ZN staining, and at least 30 low-power fields by FM with auramine staining. Cultures were performed using both LJ and MGIT. Speciation was performed on all positive cultures at the local site or at a reference laboratory. Local laboratories performed drug susceptibility testing using the BACTEC MGIT system or the Middlebrook proportion method for firstline TB drugs (rifampin, isoniazid [INH], pyrazinamide and ethambutol) or utilized a reference laboratory. Laboratory personnel were blinded to the participant symptom profile. Participants also underwent complete blood count testing and chemistry panel. Absolute CD4+ cell count and percentage assays were performed at certified laboratories. CXRs were read by local site investigators. Findings were categorized as consistent with TB if any of the following were recorded: infiltrates, cavitary lesions, miliary patterns, pleural or pericardial effusions or adenopathy. Female participants of reproductive age underwent pregnancy testing prior to CXR. CXR was performed in pregnant women if the site clinician considered that the potential benefits outweighed the potential risks, and if shielding was available.

\section{Statistical considerations}

Algorithm building was limited to participants with all key elements: symptom and physical examination questionnaires, evaluable sputum specimens and CXR. Continuous and ordered categorical variables between groups with and without TB were compared using Wilcoxon's rank-sum tests, and categorical variables between groups were compared using Fisher's exact tests. Exact binomial 95\% confidence intervals (CIs) were calculated for sensitivity and specificity. Results were not adjusted for multiple comparisons. Candidate SDE elements were analyzed separately with regard to impact on sensitivity and specificity. Model building began with the best combination from a simple symptom screen, then considered variables from the physical examination (such as lymphadenopathy), and lastly added the impact of more expensive/resource-intensive diagnostic tools such as CXR.

\section{RESULTS}

\section{Study participants}

A total of 801 persons were enrolled between 5 February and 30 November 2010 from sites in Botswana, Malawi, South Africa, Zimbabwe, India, Brazil and Peru (Figure). The median age was 33 years, and $37 \%$ of participants were male; $65 \%$ were black African, $29 \%$ Asian and $2 \%$ White. The median CD4+ cell count was 275 cells $/ \mathrm{mm}^{3}$. Ninety-one per cent $(n=$ 724) of the participants were able to produce at least one sputum specimen and $96 \%(n=$ 766) underwent CXR; a combined $87 \%(n=698)$ were able to produce sputum and also had a CXR. Eleven female participants were pregnant and did not undergo CXR. Characteristics of the 707 participants with the required data elements are given in Table 1, and reasons for exclusion are shown in the Figure.

\section{Prevalence of tuberculosis and drug resistance}

Sixteen participants had at least one positive acid-fast bacilli (AFB) smear (15 by light microscopy and 15 by FM) and 85 were culture-positive on either solid or liquid media. Of these, 55 (7.8\%, 95\% CI 5.9-10.0) were M. tuberculosis and 30 (35\%) were nontuberculous mycobacterial or unknown species. Thirty-one cultures on solid media were positive for $M$. tuberculosis and 54 on liquid media. Of the 55 participants with positive cultures, 30 were positive on both liquid and solid media, 24 were positive on liquid only and one was positive on solid media only. Collection of a third sputum specimen did not increase the yield for smear, but identified four participants by culture, providing an incremental yield of $1 \%$. The overall contamination rate was $4 \%$ for cultures on solid or liquid media. Four participants had drug-resistant TB, three to INH and one to streptomycin. All but one of the positive 
cultures were from participants at sites in sub-Saharan Africa. An analysis of data from participants without positive cultures at African versus non-African sites suggested that region would be the best predictor of TB; therefore, the remaining analyses was restricted to the 445 participants from African sites. Of the 445 Africans enrolled, 54 (12\%) had confirmed TB.

\section{Algorithm elements}

Performance of overall standard of care-Of 54 participants with TB, $49(91 \%)$ had at least one symptom of cough, fever, night sweats and weight loss; however, 308 (79\%) of 391 participants without TB also had at least one symptom. This provides $91 \%$ sensitivity (80-97), 21\% specificity (17-26) and an NPV of 94\% (87-98) among study participants.

Performance across CD4 count strata-Sensitivity for the symptom combination was $85 \%(65-96)$ and specificity $10 \%(5-17)$ in participants with a CD4 cell count $<200$ cells/ $\mathrm{mm}^{3}$, giving a positive predictive value (PPV) of 17\% (11-24) and an NPV of 75\% (48-93). In those with a CD4 cell count of $>200$ cells $/ \mathrm{mm}^{3}$, sensitivity was $96 \%$ (82-99.9) and NPV $98.6 \%$ (92-100). Of 54 participants with TB, 24 (51\%) had a normal CXR. SOC screening (symptom screen, ZN sputum smear and CXR) therefore had 54\% sensitivity (95\% CI 4067 ) and $76 \%$ specificity (95\% CI 72-80). PPV and NPV were respectively $24 \%$ (95\%CI $17-$ 32 ) and $92 \%$ (95\% CI 89-95).

Performance of standardized diagnostic evaluation elements-Performance characteristics of the individual data elements are given in Table 2. Cough in the last 30 days was commonly reported among the screened population but was more prevalent among those diagnosed with compared to those without TB $(81 \%$ vs $61 \%, P=0.003)$. Cough of $\geq$ 14 days' duration was present in $61 \%$ of those with TB and $39 \%$ of those without. In addition, the following individually all had sensitivity of $>40 \%$ : fever $(43 \%)$, night sweats $(48 \%)$, weight loss/anorexia (60/48\%), fatigue (50\%), lymphadenopathy $(67 \%)$, pulse rate > 80 beats/min (65\%), hemoglobin < $11 \mathrm{~g} / \mathrm{dl}(50 \%)$ and abnormal CXR (54\%). Five of the 54 participants with TB had none of the cardinal symptoms of TB: cough, fever, night sweats and/or weight loss. Of these, three had lymphadenopathy, and two of these three also had an abnormal CXR.

Performance characteristics of combinations of the data elements are given in Table 3. Presence of one of the cardinal symptoms with a CXR compatible with TB increased specificity to $84 \%$, but sensitivity was decreased when compared to signs and symptoms alone (44\%). Results were similar when lymphadenopathy was included in the algorithm. Fifty (93\%) participants with TB had cough or lymphadenopathy as compared to 294 (75\%) without TB $(P=0.003)$. In contrast, four $(7 \%)$ of participants with TB did not report cough or have lymphadenopathy, and 97 (25\%) of participants without TB had neither, giving $93 \%$ sensitivity (95\% CI 82-98) and 25\% specificity (95\% CI 20-29).

\section{DISCUSSION}

Our study had several important findings. First, this prospective, multi-country study validates the current WHO guidelines for symptom screening in HIV-infected persons, as $91 \%$ of patients with TB had at least one symptom. Second, inclusion of more detailed evaluation of clinical signs and symptoms did not improve sensitivity or specificity.

Of all the positive cultures, $98 \%$ were from HIV-infected persons in sub-Saharan Africa, reflecting the much higher burden of active TB at screening in this region than at the Indian and South American sites. The prevalence of previously undiagnosed TB in African sites was $12 \%$ in HIV-infected participants who were not regarded as TB suspects. Consistent 
with other studies, we found that symptom-based screening had a high NPV. ${ }^{11}$ Cough was the most sensitive symptom, particularly when combined with abnormal CXR, lymphadenopathy or a CD4 cell count of $<200$ cells $/ \mathrm{mm}^{3}$. However, smear microscopy was insufficiently sensitive to identify the majority of pulmonary TB cases, and only 6 of 54 African participants with positive cultures also had positive sputum smears. Although almost half the participants in our study with TB had normal CXR, the addition of CXR improved specificity when compared to symptom screening alone.

Limitations of our study include the use of sputum culture as the gold standard, as this is not $100 \%$ sensitive and specific. CXRs were read by site investigators rather than by using a centralized rating system. In addition, we did not perform follow-up evaluations for TB outcomes.

Our study underscores the urgent need for improved screening for TB in HIV-infected patients prior to the initiation of ART. Worldwide, TB control programs have dramatically improved rates of HIV screening of TB suspects, but rapid, low-cost point-of-care screening tests for HIV infection have excellent performance characteristics. ${ }^{19}$ In contrast, integration of TB screening into ART programs presents many challenges. Symptom-based screening has excellent NPV, particularly for identifying patients who may benefit from INH preventive therapy. ${ }^{12}$ However, the current reliance on smear microscopy to diagnose TB is insufficiently sensitive, and symptomatic HIV-infected patients should be tested with more sensitive tests, such as culture and/or nucleic acid amplification tests. Moreover, recent reports of multi- and extensively drug-resistant TB have heightened concerns about missed diagnoses and inadequate treatment for $\mathrm{TB} .{ }^{20}$ These findings further emphasize the need for rapid molecular tests for detection of $\mathrm{TB}$ and drug resistance at the point of care to implement appropriate treatment.

\section{Supplementary Material}

Refer to Web version on PubMed Central for supplementary material.

\section{Acknowledgments}

The authors thank study team investigators: R Chaisson, Johns Hopkins University Baltimore MD, USA, and S Pillay, University of KwaZulu-Natal, Durban, South Africa; P Kim, Medical Officer, Division of Acquired Immunodeficiency Syndrome, National Institute of Allergy and Infectious Diseases (NIAID), National Institutes of Health (NIH), V Kumar, Mycobacteriologist Tuberculosis Research Centre, Chetput, Chennai, India, and P Coulter, Johns Hopkins University/Patient Safety Monitoring in International Laboratories, Baltimore, MD, USA (laboratory consultants); B Putnam, University of Colorado Health Sciences Center, Denver, CO, USA (field representative); K Buck, Frontier Science \& Technology Research Foundation, Amherst, NY, USA (data manager); N Carpenti, College of Medicine-Johns Hopkins Research Project Laboratory, Blantyre, Malawi, and L MongKryspin (American Society for Clinical Pathology) The Ohio State University, Columbus, OH, USA (laboratory technicians); A Manzella, Frontier Science and Technology Research Foundation, Amherst, NY, USA (laboratory data coordinator); S Warner and C Blanchard-Horan PhD, ACTG Operations Center, Silver Spring, MD, USA (International Program Specialists); and P Ndhleni Ziba, Lusaka, Zambia (Network Community Advisory Board Representative). Compensation was not received by these individuals.

This work was supported by NIH grants U01AI068636 from the NIAID; U01 AI068634 for the Statistical and Data Management Center for the AIDS Clinical Trials Group; and AI51519 for the Center for AIDS Research at the Albert Einstein College of Medicine (supplement for JMA). This study was presented in part at the $19^{\text {th }}$ Conference on Retroviruses and Opportunistic Infections, Seattle, Washington, USA, 5-8 March 2012.

\section{References}

1. World Health Organization. Global Plan to Stop TB. Geneva, Switzerland: WHO; 2006. Stop TB Partnership. 
2. Chaisson RE, Martinson NA. Tuberculosis in Africa--combating an HIV-driven crisis. N Engl J Med. 2008; 358:1089-1092. [PubMed: 18337598]

3. Bassett IV, Wang B, Chetty S, et al. Intensive tuberculosis screening for HIV-infected patients starting antiretroviral therapy in Durban, South Africa. Clin Infect Dis. 2010; 51:823-829.

[PubMed: 20735240]

4. Coetzee D, Hilderbrand K, Goemaere E, Matthys F, Boelaert M. Integrating tuberculosis and HIV care in the primary care setting in South Africa. Trop Med Int Health. 2004; 9:A11-15. [Abstract]. [PubMed: 15189469]

5. Centers for Disease Control and Prevention. Atlanta, GA, USA: CDC; Tuberculosis (TB) (Mycobacterium tuberculosis) 2009 case definition. http://wwwn.cdc.gov/NNDSS [Accessed January 2013]

6. Lawn SD, Bekker LG, Middelkoop K, Myer L, Wood R. Impact of HIV infection on the epidemiology of tuberculosis in a peri-urban community in South Africa: the need for age-specific interventions. Clin Infect Dis. 2006; 42:1040-1047. [PubMed: 16511773]

7. Scott LE, McCarthy K, Gous N, et al. Comparison of Xpert MTB/RIF with other nucleic acid technologies for diagnosing pulmonary tuberculosis in a high HIV prevalence setting: a prospective study. PLoS Med. 2011; 8:e1001061. [PubMed: 21814495]

8. Young DB, Perkins MD, Duncan K, Barry CE III. Confronting the scientific obstacles to global control of tuberculosis. Journal Clin Invest. 2008; 118:1255-1265.

9. Dalton T, Cegielski P, Akksilp S, et al. Prevalence of and risk factors for resistance to second-line drugs in people with multidrug-resistant tuberculosis in eight countries: a prospective cohort study. Lancet. 2012; 380:1406-1417. [PubMed: 22938757]

10. Getahun H, Harrington M, O’Brien R, Nunn P. Diagnosis of smear-negative pulmonary tuberculosis in people with HIV infection or AIDS in resource-constrained settings: informing urgent policy changes. Lancet. 2007; 369:2042-2049. [PubMed: 17574096]

11. Cain KP, McCarthy KD, Heilig CM, et al. An algorithm for tuberculosis screening and diagnosis in people with HIV. N Engl J Med. 2010; 362:707-716. [PubMed: 20181972]

12. Getahun H, Kittikraisak W, Heilig CM, et al. Development of a standardized screening rule for tuberculosis in people living with HIV in resource-constrained settings: individual participant data meta-analysis of observational studies. PLoS Med. 2011; 8:e1000391. [PubMed: 21267059]

13. World Health Organization. Guidelines for intensified tuberculosis case-finding and isoniazid preventive therapy for people living with HIV in resource-constrained settings. Geneva, Switzterland: WHO; 2011.

14. World Health Organization. Global health atlas. Geneva, Switzerland: WHO; 2003-2007. http:// www.who.int/globalatlas [Accessed January 2013]

15. Mase SR, Ramsay A, Ng V, et al. Yield of serial sputum specimen examinations in the diagnosis of pulmonary tuberculosis: a systematic review. Int J Tuberc Lung Dis. 2007; 11:485-495. [PubMed: 17439669]

16. Kent, P.; Kubica, GP. A guide for level III laboratory. Atlanta, Georgia: US Department of Healtn and Human Services, Centers for Disease Control and Prevention; 1985. Public health mycobacteriology.

17. World Health Organization. Part II: microscopy. Geneva, Switzerland: WHO; 1998. Laboratory services in tuberculosis control. WHO/TB/98.258

18. International Union Against Tuberculosis and Lung Disease. Technical guide. 5. Paris, France: The Union; 2000. Sputum examination for tuberculosis by direct microscopy in low income countries.

19. Pai NP, Balram B, Shivkumar S, et al. Head-to-head comparison of accuracy of a rapid point-ofcare HIV test with oral versus whole-blood specimens: a systematic review and meta-analysis. Lancet Infect Dis. 2012; 12:373-380. [PubMed: 22277215]

20. Andrews JR, Shah NS, Weissman D, Moll AP, Friedland G, Gandhi NR. Predictors of multidrugand extensively drug-resistant tuberculosis in a high HIV prevalence community. PloS ONE. 2010; 5:e15735. [PubMed: 21209951] 


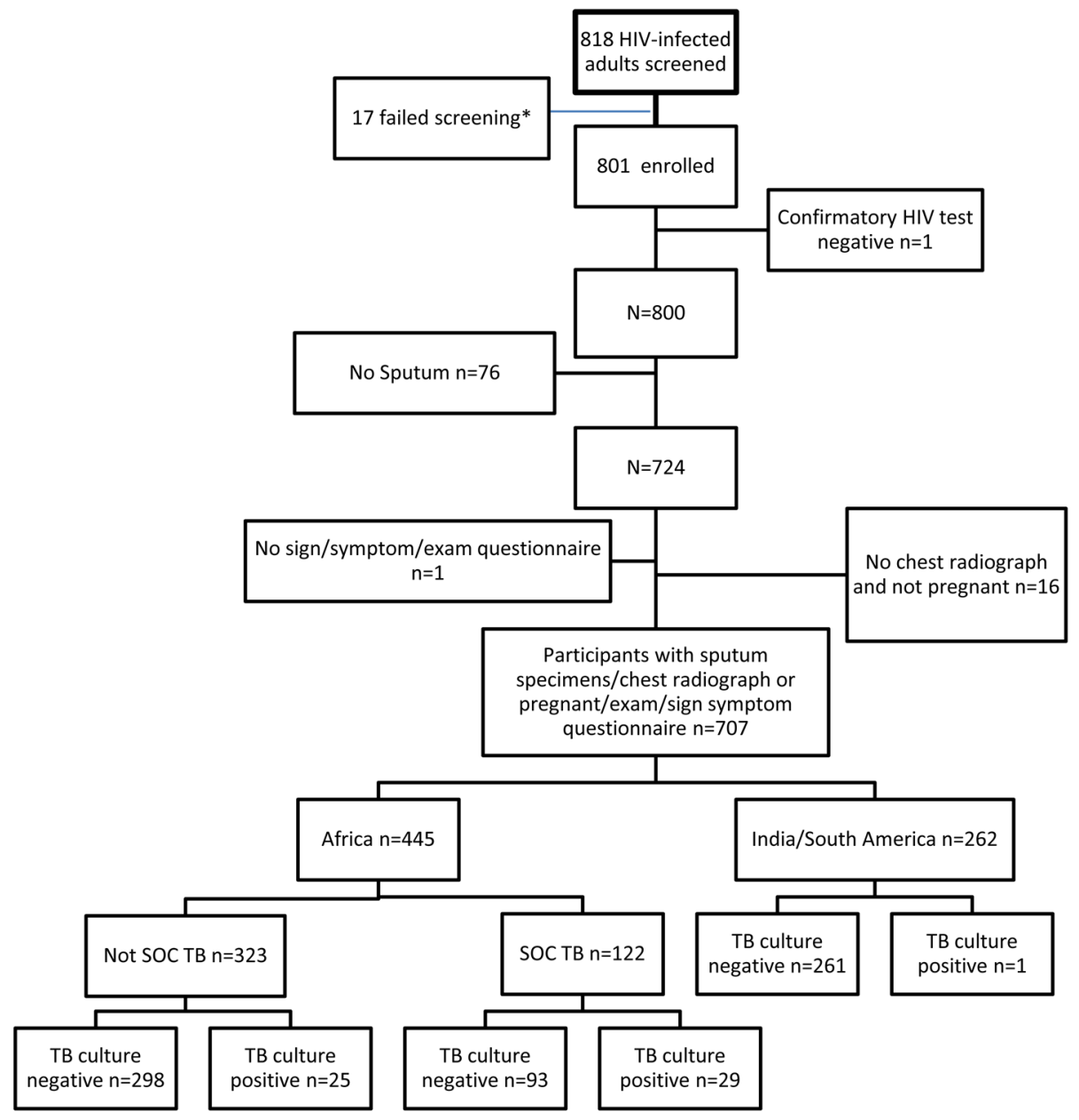

Figure.

*Reasons for screen failures were: 9 were assigned 2 screening numbers; 3 failed appointments; 1 was taking prohibited medication; 1 negative HIV test; 1 unable to produce sputum; 2 screened after accrual was complete 


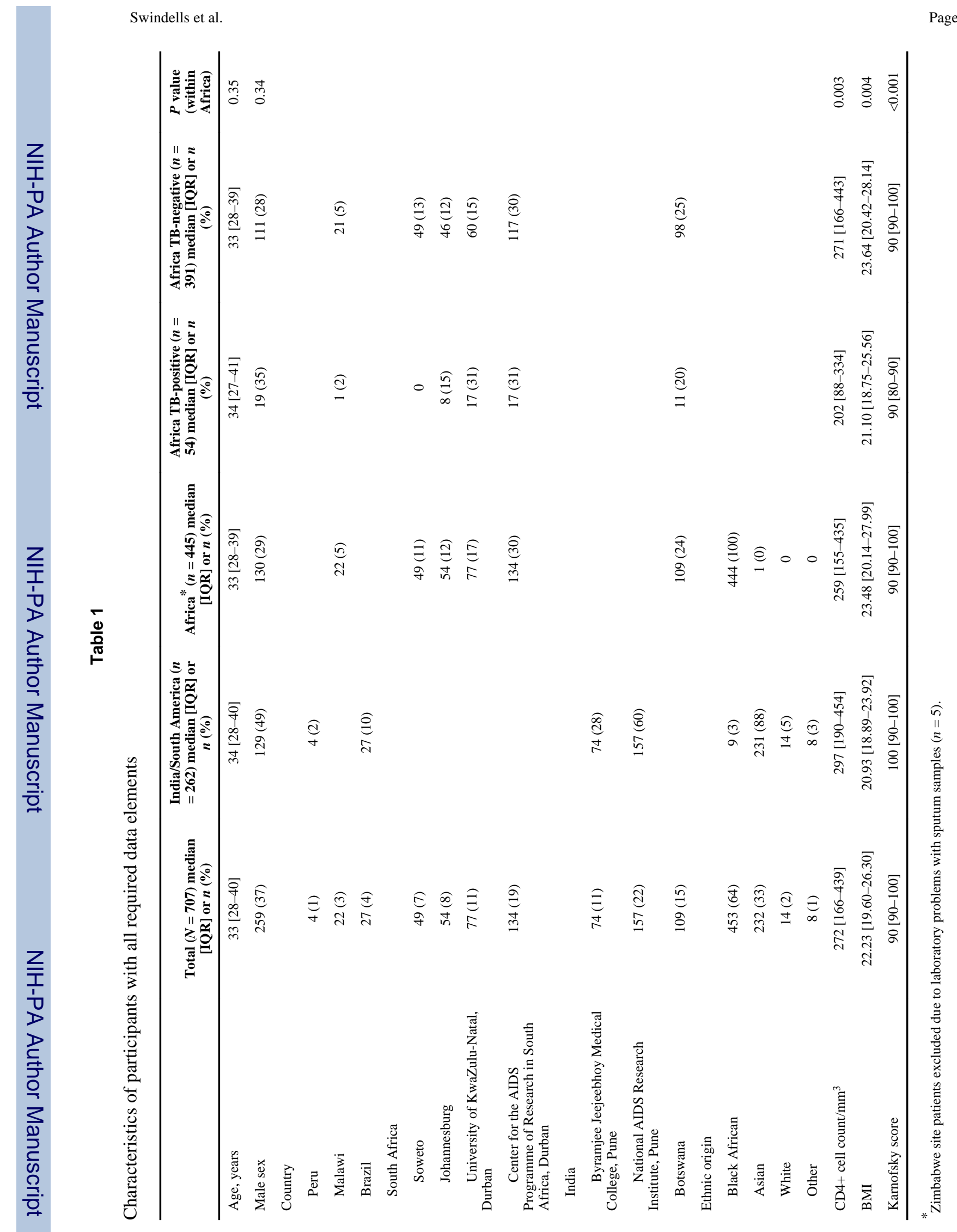

Int J Tuberc Lung Dis. Author manuscript; available in PMC 2014 February 13. 


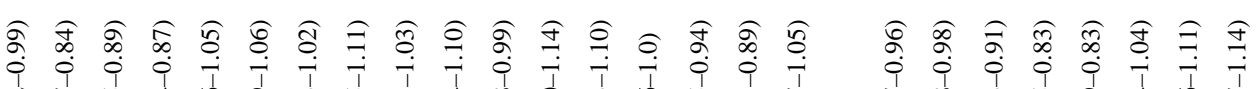

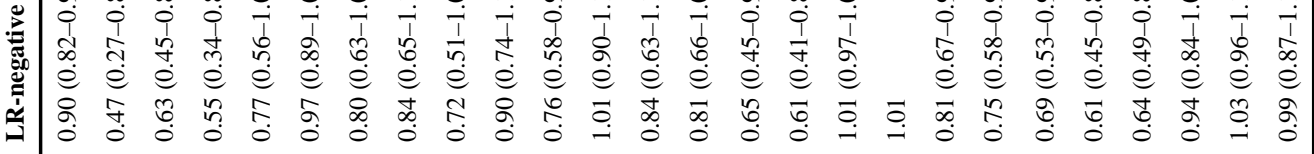

क人ष

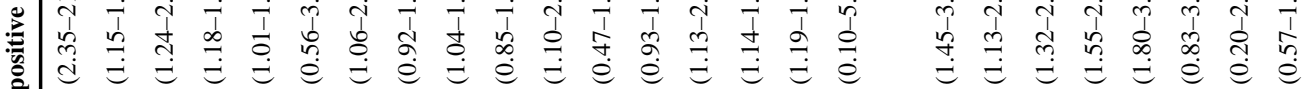

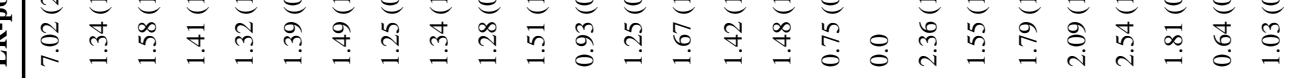

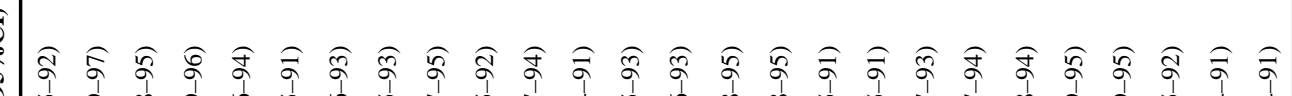

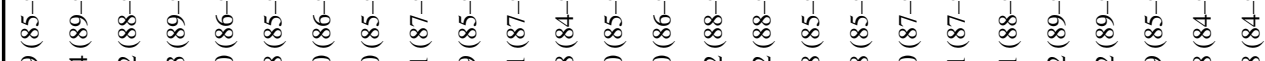

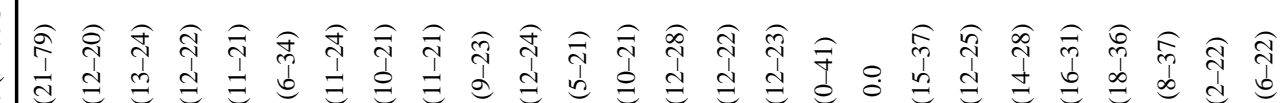

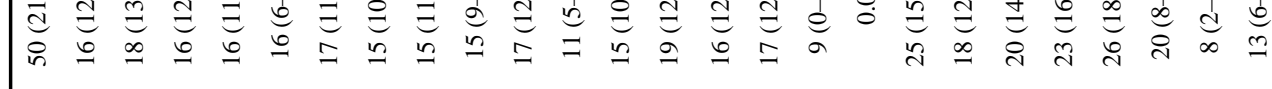

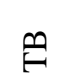

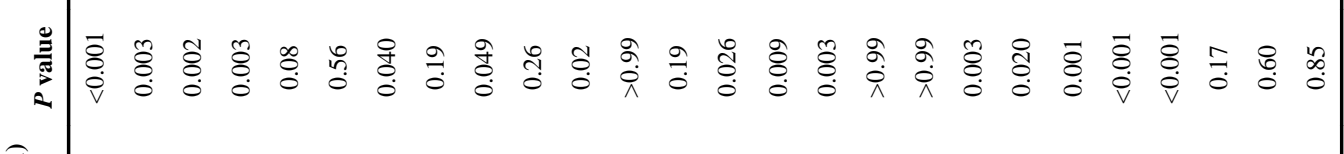

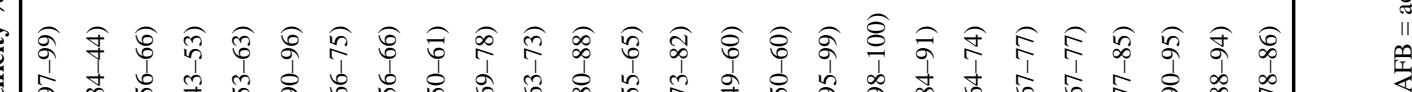

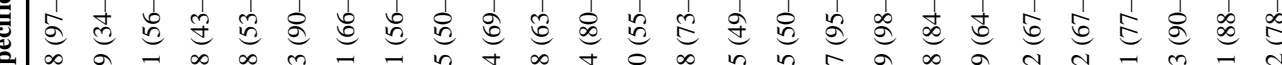

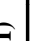

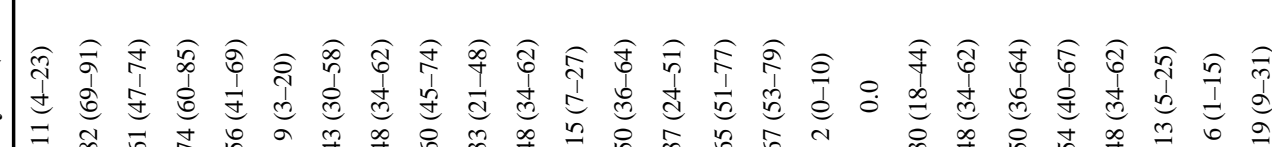

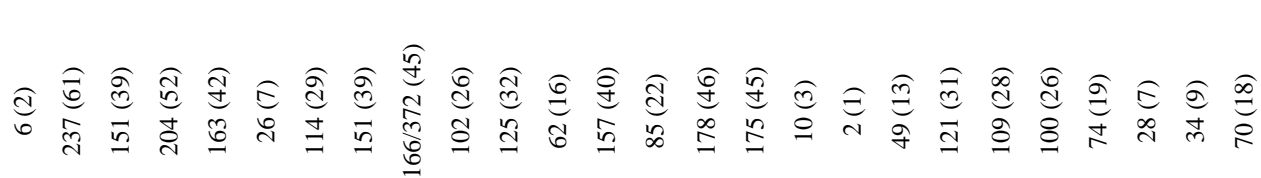

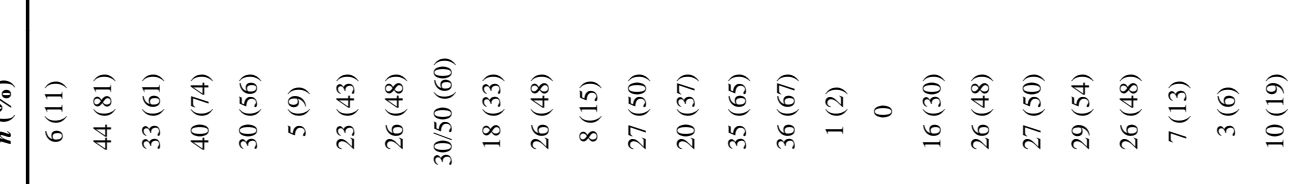


